\title{
Periodontal Powder Dosage Form
}

National Cancer Institute

\section{Source}

National Cancer Institute. Periodontal Powder Dosage Form. NCI Thesaurus. Code C149752.

Solid preparation consisting of one or more powders intended for administration within the tooth socket/periodontal membrane. 